\title{
TIDAL NOVAE IN COMPACT BINARY WHITE DWARFS
}

\author{
Jim Fuller and Dong LaI \\ Department of Astronomy, Cornell University, Ithaca, NY 14850, USA \\ Received 2012 June 3; accepted 2012 July 30; published 2012 August 14
}

\begin{abstract}
Compact binary white dwarfs (WDs) undergoing orbital decay due to gravitational radiation can experience significant tidal heating prior to merger. In these WDs, the dominant tidal effect involves the excitation of outgoing gravity waves in the inner stellar envelope and the dissipation of these waves in the outer envelope. As the binary orbit decays, the WDs are synchronized from outside in (with the envelope synchronized first, followed by the core). We examine the deposition of tidal heat in the envelope of a carbon-oxygen WD and study how such tidal heating affects the structure and evolution of the WD. We show that significant tidal heating can occur in the star's degenerate hydrogen layer. This layer heats up faster than it cools, triggering runaway nuclear fusion. Such "tidal novae" may occur in all WD binaries containing a CO WD, at orbital periods between 5 minutes and 20 minutes, and precede the final merger by $10^{5}-10^{6}$ years.
\end{abstract}

Key words: binaries: close - hydrodynamics - novae, cataclysmic variables - white dwarfs

Online-only material: color figures

\section{INTRODUCTION}

Compact white dwarf (WD) binaries (with orbital periods in the range from minutes to hours) are important for several areas of astrophysics. The orbits of these systems decay via the emission of gravitational waves, constituting the largest signals for the next generation of space-based gravitational wave interferometers. Systems of sufficiently short orbital period will merge within a Hubble time, the result of which may produce a variety of exotic objects, such as helium-rich sd0 stars, R CrB stars, and AM CVn binaries. Most importantly, when the total binary mass is near the Chandrasekhar limit, the merged WDs may collapse into a neutron star or explode as a Type Ia supernova (SN Ia; e.g., Webbink 1984; Iben \& Tutukov 1984). Recent studies have provided support for such "double degenerate" progenitors of SNe Ia. (e.g., Gilfanov \& Bogdan 2010; Di Stefano 2010; Maoz et al. 2010; Li et al. 2011; Bloom et al. 2012; Schaefer \& Pagnotta 2012).

The outcome of a WD binary merger depends on the masses of the WDs and their pre-merger conditions (e.g., Segretain et al. 1997; Yoon et al. 2007; Loren-Aguilar et al. 2009; van Kerkwijk et al. 2010; Dan et al. 2012; Raskin et al. 2012). Most previous studies of pre-merger binary WDs have focused on equilibrium tides and considered tidal dissipation in a parameterized way (e.g., Mochkovitch \& Livio 1989; Iben et al. 1998; Willems et al. 2010; Piro 2011). None of these studies have sought to predict the magnitude and location of tidal heating due to dynamical tides, which dominate the tidal responses of the binary WDs.

In two recent papers (Fuller \& Lai 2011, 2012, hereafter Paper I and Paper II, respectively), we presented the first ab initio calculations of dynamical tides in realistic WD models. In Paper I, we considered resonant excitations of WD $g$-modes during binary decay and showed that the modes reach nonlinear amplitudes near the surface of the star. This implies that, rather than exciting discrete $g$-modes, the binary companion will excite a continuous train of gravity waves, which propagate outward and dissipate in the outer envelope of the WD. We studied such continuous tidally excited waves in Paper II. For a canonical carbon/oxygen WD (consisting of $\mathrm{CO}$ core with an $\mathrm{He}-\mathrm{H}$ envelope), we showed that the outgoing waves are primarily launched at the $\mathrm{CO} / \mathrm{He}$ transition region, and propagate toward the WD surface, where they are likely dissipated through a combination of non-linear processes and radiative damping. We computed the energy and angular momentum flux carried by the waves in order to predict the orbital and spin evolution of WDs in compact binaries. We found that such dynamical tides cause the binary WDs to be nearly synchronized prior to merger. Furthermore, the tidal heating rate can be quite large at short orbital periods (exceeding tens of solar luminosities just before merger, depending on the system parameters), potentially leading to significant observable signatures.

In this Letter, we show that tidal heating may trigger a thermonuclear runaway hydrogen fusion event in a CO WD. The observational consequence of such an event would likely be an outburst that resembles a classical nova. We call this new phenomenon a "tidal nova" (TN). Unlike all other types of novae or supernovae, a TN does not rely on mass accretion or collapse. We present a simple two-zone model for the angular momentum evolution of a differentially rotating WD, which we use to calculate the radial tidal heating profile within the WD. We then evolve the WD model including tidal heating to calculate changes in its temperature, luminosity, and internal structure. For a wide range of physically plausible parameters, we demonstrate that tidal heating induces a thermonuclear runaway event. Finally, we discuss the observational signatures of such an event, and compare our predictions to observations of short-period WD binaries.

\section{ENERGY AND ANGULAR MOMENTUM OF TIDALLY EXCITED GRAVITY WAVES}

Using the method described in Paper II, we calculate the amplitude of tidally excited gravity waves inside a WD. We consider a circular orbit with angular frequency $\Omega$. The WD spins at an angular frequency $\Omega_{s}$, and the spin is aligned with the orbit. In the corotating frame, the frequency of the dominant $l=m=2$ tidal potential is $\omega=2\left(\Omega-\Omega_{s}\right)$. For a WD of mass $M$ and radius $R$ (and given internal structure) with a companion of mass $M^{\prime}$, the energy and angular momentum fluxes carried 
by the gravity waves can be written as

$$
\begin{gathered}
\dot{J}_{z}(\Omega, \omega)=T_{0}(\Omega) F(\omega), \\
\dot{E}(\Omega, \omega)=\Omega T_{0} F(\omega),
\end{gathered}
$$

where

$$
T_{0}(\Omega)=\frac{G M^{\prime 2}}{a}\left(\frac{R}{a}\right)^{5},
$$

with $\Omega=\sqrt{G M_{t} / a^{3}}$ the orbital angular frequency $\left(M_{t}=\right.$ $M+M^{\prime}$ is the total mass and $a$ is the orbital semimajor axis).

The dimensionless function $F(\omega)$ (similar to the tidal lag angle in the language of equilibrium tide theory) determines the magnitude of wave excitation and is strongly dependent on the internal structure of the WD and the tidal frequency $\omega$. In Paper II, we have calculated $F(\omega)$ for $0.6 M_{\odot}$ CO WD models of various surface temperatures and slow rotation. We found that $F(\omega)$ is an erratic function of $\omega$ because of the "quasiresonance cavity" formed by the $\mathrm{CO}$ core inside the $\mathrm{He} / \mathrm{H}$ shell. However, because of the strong dependence of $F(\omega)$ on $\omega$ (the envelope of $F(\omega)$ approximately scales as $\omega^{5}$ ), at sufficiently short orbital periods, tidal spin-up combined with orbital decay via gravitational radiation ensure that $\omega \simeq$ const. The orbital period at which this transition occurs is $P_{c} \simeq 40$ minutes, depending on the WD masses and temperatures (see Equation (79) of Paper II). At periods $P \lesssim P_{c}$, the tidal energy transfer rate is

$$
\dot{E} \simeq \frac{3 I \Omega^{2}}{2 t_{\mathrm{GW}}}
$$

where $I$ is the moment of inertia of the $\mathrm{WD}$, and $t_{\mathrm{GW}}=|a / \dot{a}|$ is the binary inspiral time due to gravitational radiation,

$$
t_{\mathrm{GW}}=4.2 \times 10^{5} \mathrm{yr}\left(\frac{M_{\odot}^{2}}{M M^{\prime}}\right)\left(\frac{M_{t}}{2 M_{\odot}}\right)^{1 / 3}\left(\frac{P}{10 \text { minutes }}\right)^{8 / 3}
$$

When the outgoing gravity waves damp in the WD envelope and locally deposit their angular momentum, some of the wave energy is converted into rotational kinetic energy, while the rest is converted to heat. The heating rate is

$$
\dot{E}_{\text {heat }}=\dot{E}\left(1-\frac{\Omega_{s}}{\Omega}\right) \text {. }
$$

If the WD maintains some differential rotation, $\Omega_{s}$ in the above equation should be the rotation rate of the layer in which the waves damp, and heat will also be generated through viscous angular momentum transport.

\section{TWO-ZONE MODEL FOR TIDAL HEAT DEPOSITION}

Our calculations indicate that the gravity waves reach nonlinear amplitudes and break in the outer layers of the WD. The location of wave breaking depends on various parameters (e.g., orbital and tidal frequencies), but is always at $r \gtrsim 0.92 R$ and the exterior mass $\Delta M \lesssim 10^{-4} M$ (Paper II). Since a small fraction of the stellar mass absorbs the entire angular momentum flux, the outer layer spins up rapidly. If it spins up faster than angular momentum can be transported to the core, the outer layer will rotate synchronously with the orbit. Outgoing waves approaching the synchronized envelope will be absorbed near corotation and deposit their angular momentum, causing the synchronized envelope to move to larger depths (see Goldreich \& Nicholson 1989).

We consider a simple two-zone model for the spin evolution of the WD. In this model, the envelope of the star rotates synchronously with the orbit $\left(\Omega_{\mathrm{env}}=\Omega\right)$, while the core rotates sub-synchronously $\left(\Omega_{\text {core }}<\Omega\right)$. The envelope and core are coupled, with angular momentum being transferred to the core according to a parameterized coupling time, $t_{\text {coup }}$. The angular momentum of the core-envelope system evolves according to

$$
\begin{gathered}
\frac{d}{d t}\left(I_{\mathrm{env}} \Omega_{\mathrm{env}}\right)=\dot{J}_{z}\left(\Omega, \omega_{\text {core }}\right)-\frac{I_{\mathrm{env}}}{t_{\text {coup }}}\left(\Omega_{\mathrm{env}}-\Omega_{\mathrm{core}}\right), \\
\frac{d}{d t}\left(I_{\text {core }} \Omega_{\text {core }}\right)=\frac{I_{\mathrm{env}}}{t_{\text {coup }}}\left(\Omega_{\mathrm{env}}-\Omega_{\text {core }}\right)
\end{gathered}
$$

where $I_{\text {env }}=I-I_{\text {core }}$ is the moment of inertia of the envelope. Here, $\dot{J}_{z}$ is the angular momentum flux which can be calculated from Equation (1). We have assumed that the gravity waves are excited in the core and absorbed in the envelope. ${ }^{1}$ Consequently, the angular momentum source term $\dot{J}_{z}$ is only present in the envelope evolution equation, although it is dependent on the tidal frequency in the core, $\omega_{\text {core }}=2\left(\Omega-\Omega_{\text {core }}\right)$. Using $\Omega_{\text {env }}=\Omega$, Equations (7) and (8) can be integrated to find $I_{\text {env }}$ and $\Omega_{\text {core }}$ as a function of time or orbital period. The mass $\Delta M_{\text {env }}$ of the envelope corresponds to $I_{\text {env }} \simeq(2 / 3) \Delta M_{\text {env }} R^{2}$.

The thickness (or $\Delta M_{\text {env }}$ ) of the envelope is dependent

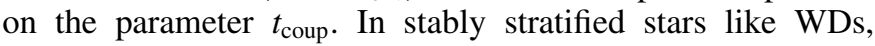
angular momentum can be transported by magnetic fields. In the presence of a poloidal field $B$ connecting the core and envelope, $t_{\text {coup }}$ can be estimated from the Alfvén wave crossing time

$$
t_{A}=\frac{R \sqrt{4 \pi \rho}}{B} \simeq 10^{2} \operatorname{yr}\left(\frac{10^{3} \mathrm{G}}{B}\right)
$$

for our CO WD model. For WDs without an intrinsic magnetic field, angular momentum may be transported via the Tayler-Spruit dynamo (Spruit 2002). To estimate $t_{\text {coup }}$, we calculate the effective viscosity for angular momentum transport via the Tayler-Spruit dynamo, $\nu_{\mathrm{TS}}$, as outlined in Spruit (2002). ${ }^{2}$ We find $t_{\mathrm{TS}} \equiv \int_{0}^{R}\left(r / \nu_{\mathrm{TS}}\right) d r \approx 10^{3} \operatorname{yr}(P / 45 \text { minutes })^{3 / 2}$. Thus, we expect the coupling time to lie in the range $t_{\text {coup }} \lesssim 10^{3} \mathrm{yr}$ for the short orbital periods of interest.

Figure 1 plots the value of $\Delta M_{\mathrm{env}}$ as a function of orbital period for our $0.6 M_{\odot}$ WD model with a $0.3 M_{\odot}$ companion, using values of $t_{\text {coup }}$ ranging from $1 \mathrm{yr}$ to $10^{3} \mathrm{yr}$. We begin our calculation at $P_{\text {orb }}>1 \mathrm{hr}$ and use $I_{\mathrm{env}, 0}=0$ and $\Omega_{\text {core }, 0}=0$, as appropriate at long orbital periods where tidal effects are negligible. We see that for the range of $t_{\text {coup }}$ considered, $\Delta M_{\mathrm{env}}$ remains small $\left(\lesssim 10^{-2} M_{\odot}\right)$ at all orbital periods of interest. Thus, the synchronized envelope most likely does not extend down to the $\mathrm{C} / \mathrm{He}$ transition layer where the gravity waves are excited, justifying our assumption that $\dot{J}_{z}$ is a function of $\Omega_{\text {core }}$. However, the envelope does extend to very large optical depths, suggesting that binary WDs may be observed to be synchronized at large orbital periods even if their cores are not synchronized.

\footnotetext{
1 This assumption is valid as long as long as the core-envelope boundary is above the $\mathrm{C} / \mathrm{He}$ transition layer (with an exterior mass $\Delta M \approx 10^{-2} M_{\odot}$ ), which is the region where the outgoing gravity waves are excited. 2 For simplicity, we have calculated the viscosity $v_{\mathrm{TS}}$ without including the effects of composition gradients in the WD (see Equation (32) in Spruit 2002). A more realistic estimate of the rotational profile of the WD should take composition gradients into account.
} 


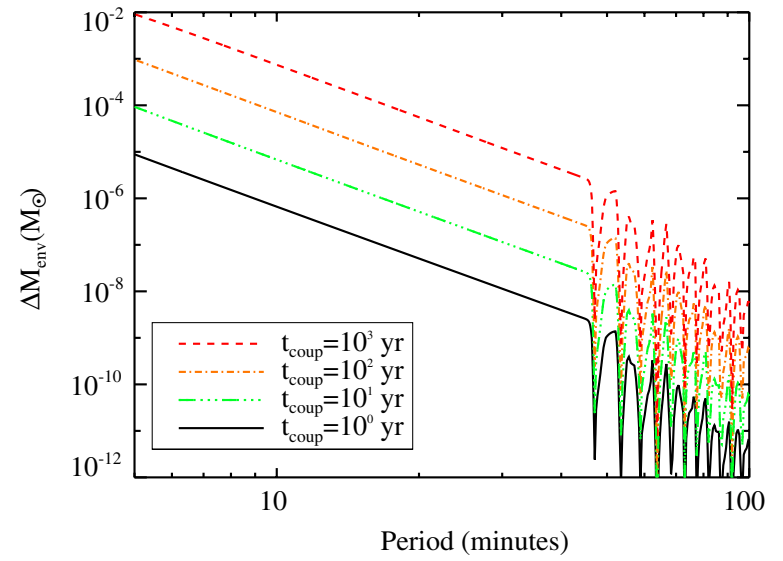

Figure 1. Mass $\Delta M_{\mathrm{env}}$ of the synchronized envelope as a function of orbital period for a $0.6 M_{\odot}$ CO WD model with a $0.3 M_{\odot}$ companion. The solid (black) line has $t_{\text {coup }}=1 \mathrm{yr}$, the dot-dot-dashed (green) line $t_{\text {coup }}=10 \mathrm{yr}$, the dot-dashed (orange) line $t_{\text {coup }}=10^{2} \mathrm{yr}$, and the dashed (red) line $t_{\text {coup }}=10^{3} \mathrm{yr}$. (A color version of this figure is available in the online journal.)

Note that since $I_{\mathrm{env}} \ll I$, the core of the star contains most of the angular momentum, and its spin evolves in the same manner as discussed in Paper II.

\section{TIDAL HEATING AND UNSTABLE NUCLEAR BURNING}

In the two-zone model discussed in Section 3, the total tidal heating rate $\dot{E}_{\text {heat }}$ may be calculated from Equation (6) with $\Omega_{s}=\Omega_{\text {core }}$, and the tidal heat is deposited entirely at the base of the synchronized envelope where $\Delta M=\Delta M_{\text {env }}$. In a real WD, the heat deposition will occur over a range of depths that depends on the details of wave breaking and viscous angular momentum transport. For simplicity, here we choose to deposit the tidal heat uniformly per unit mass in the synchronized envelope. The heating rate per unit mass, $\dot{\varepsilon}_{\text {heat }}$, is then

$$
\begin{aligned}
& \dot{\varepsilon}_{\text {heat }}=0 \text { for } \quad \Delta M>\Delta M_{\text {env }} \\
& \dot{\varepsilon}_{\text {heat }}=\frac{\dot{E}_{\text {heat }}}{\Delta M_{\text {env }}} \text { for } \Delta M<\Delta M_{\text {env }} .
\end{aligned}
$$

Although the radial dependence of this heating function is unlikely to be realistic, we find that the results below are not strongly dependent on the form of $\dot{\varepsilon}_{\text {heat }}$.

To understand the effect of tidal heating on the WD properties, we evolve WD models using the extra heating term calculated via Equation (10). We use the one-dimensional stellar evolution code MESA (Paxton et al. 2011) to evolve our WD models, starting from an initial orbital period of $1 \mathrm{hr}$. We present results for a $0.6 M_{\odot} \mathrm{CO}$ WD model with a $\sim 10^{-4} M_{\odot}$ hydrogen shell and a $0.3 M_{\odot}$ companion.

Figure 2 displays the surface temperature as a function of orbital period for our tidally heated WD. For comparison, we also show the temperature of a non-tidally heated WD and the "tidal heating temperature," defined as

$$
T_{\text {eff,heat }}=\left(\frac{\dot{E}_{\text {heat }}}{4 \pi R^{2} \sigma}\right)^{1 / 4} .
$$

At long orbital periods ( $P \gtrsim 45$ minutes), the tidal heating has little effect on the surface temperature of the WD. At shorter periods ( $P \lesssim 30$ minutes), the temperature becomes

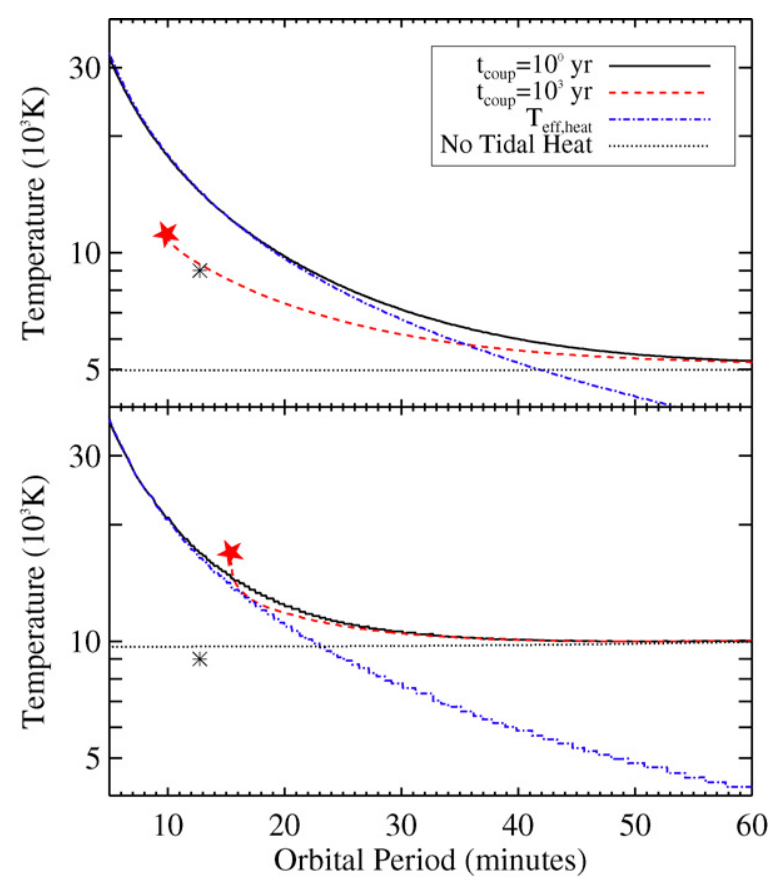

Figure 2. Surface temperature of the $0.6 M_{\odot} \mathrm{CO}$ WD model with a $0.3 M_{\odot}$ companion as a function of orbital period, for initial temperatures of $5000 \mathrm{~K}$ (top) and $10^{4} \mathrm{~K}$ (bottom). The solid black lines are calculated with $t_{\text {coup }}=1 \mathrm{yr}$ while the dashed (red) lines are calculated with $t_{\text {coup }}=10^{3} \mathrm{yr}$. The dotted lines are calculated for a WD with no tidal heating and the same initial temperature. The (blue) dot-dashed lines correspond to Equation (11). The (red) stars mark the points at which tidal novae occur. The asterisks mark the position of the secondary of the 12.75 minute binary WD system SDSS J065133+284423 (Brown et al. 2011).

(A color version of this figure is available in the online journal.)

substantially larger due to tidal heating. Several of the curves end abruptly due to the ignition of a thermonuclear runaway event, at which point we terminate our evolution calculations.

For small values of $t_{\text {coup }}$, the tidal heat is deposited at shallow depths and quickly diffuses to the surface such that the luminosity of the WD is $L \simeq L_{0}+\dot{E}_{\text {heat }}$, where $L_{0}$ is the luminosity of a non-tidally heated WD. However, for larger values of $t_{\text {coup }}$, most of the tidal heat is deposited deeper in the WD where it cannot quickly diffuse outward. This leads to lower surface temperatures, although the internal temperature may increase substantially.

Figure 3 shows the interior temperature profile of our WD at three different orbital periods, using $t_{\text {coup }}=10^{3} \mathrm{yr}$. At long orbital periods, the temperature profile is similar to that of a nontidally heated WD. As the orbital period decreases, the interior heats up, with the local temperature maximum at $\Delta M \sim \Delta M_{\mathrm{env}}$. If the base of the hydrogen layer reaches a temperature of $\sim 10^{7} \mathrm{~K}$, hydrogen burning will be ignited.

In the depicted model, the layer just above the $\mathrm{He} / \mathrm{H}$ transition (at $\Delta M \approx 10^{-4} M_{\odot}$ ) is composed of largely degenerate hydrogen gas. The ignition of fusion in this layer can thus spark a thermonuclear runaway. In general, our calculations show that these tidal novae occur only in initially cool WDs $\left(T_{\text {eff }} \lesssim 1.2 \times 10^{4} \mathrm{~K}\right.$ in the absence of tidal heating). They do not occur in hotter WDs because the hydrogen is not degenerate and can burn stably. Also, TNe require that the waves deposit some of the heat near the base of the hydrogen layer, i.e., $10^{-5} M_{\odot} \lesssim \Delta M_{\text {env }} \lesssim 10^{-3} M_{\odot}$. In our two-zone model, such heating occurs for coupling times $10 \mathrm{yr} \lesssim t_{\text {coup }} \lesssim 10^{4} \mathrm{yr}$. Overall, we find that the TNe occur at orbital periods 5 minutes $\lesssim P_{\text {orb }} \lesssim 20$ minutes, depending on 


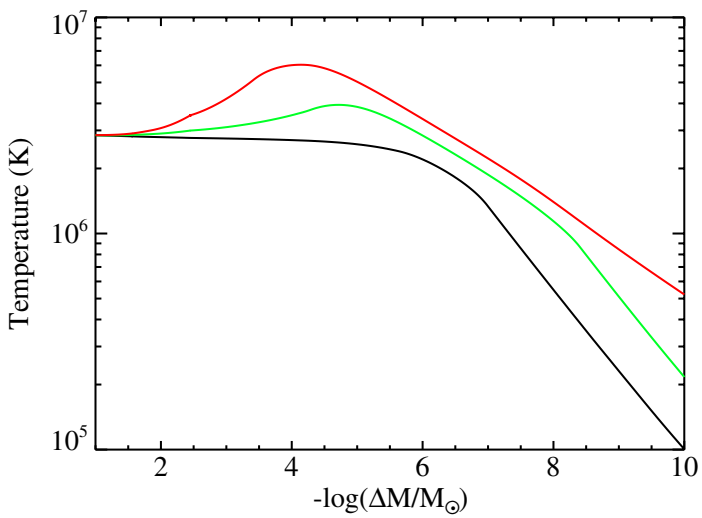

Figure 3. Temperature profile of the WD (as a function of exterior mass $\Delta M$ ) at orbital periods of 45 minutes (black), 20 minutes (green), and 12 minutes (red). These temperatures are calculated for the $0.6 M_{\odot}$ WD model with an initial surface temperature of $T_{\text {eff }}=5000 \mathrm{~K}$, a $0.3 M_{\odot}$ companion, and $t_{\text {coup }}=10^{3} \mathrm{yr}$. (A color version of this figure is available in the online journal.)

the location of heat deposition, initial temperature of the WD, and companion mass.

\section{DISCUSSION}

We have shown that under rather general conditions (see the last paragraph of Section 4), tidal dissipation in compact WD binaries can lead to nova outbursts prior to binary merger or mass transfer. While we do not attempt to predict the detailed observational signal of a $\mathrm{TN}$, we speculate that it may be very similar to a classic nova. However, in contrast to classical novae in CVs, a TN would occur in a compact system with no evidence for mass transfer. Our results indicate that a TN would precede the beginning of mass transfer or merger by about $t_{\mathrm{GW}} / 4 \sim 10^{5}-10^{6} \mathrm{yr}$ (see Equation (5)), provided the conditions outlined in the previous paragraph are satisfied.

In most classical novae, the initial outburst is followed by a period of stable hydrogen burning near the Eddington luminosity, in which the hydrogen shell of the WD inflates to a radius of order $R_{\odot}$. However, the ultracompact nature of the WD system involved in a TN (where $a \sim R_{\odot} / 4$ ) may preclude such a phase because the stably burning hydrogen shell would inflate beyond the WD's Roche lobe. This shell may then accrete onto the companion star or be ejected from the system. Therefore, we expect most of the hydrogen to be burned or ejected during in a $\mathrm{TN}$. In the absence of mass transfer to supply fresh hydrogen, recurrent novae would be unlikely. Thus, the occurrence rate of these TNe may be comparable to that of WD mergers involving a CO WD.

Our theory can be constrained by comparing the prediction of our tidal heating calculations to observed compact WD binaries. The 12.75 minute system SDSS JJ065133+284423 provides the best opportunity (Brown et al. 2011). This system is composed of a primary with $T_{\text {eff }}=16400 \mathrm{~K}$ and mass $0.25 M_{\odot}$, and a secondary with $T_{\text {eff }} \approx 9000 \mathrm{~K}$ and mass $0.55 M_{\odot}$. Comparison with Figure 2 indicates that the luminosity of the secondary is likely dominated by tidal heating. Our result for a CO WD with an initial temperature of $5000 \mathrm{~K}$ and a value of $t_{\text {coup }}=10^{3} \mathrm{yr}$ is most consistent with the observed temperature of the secondary. These results indicate that a TN may occur in this system in the future.

In principle, tidal heating may change the structure of the WD enough to alter the dynamics of gravity wave propagation. However, we find that this is not the case (i.e., no interior convection zone forms), with the exception of a thermonuclear runaway event. Our simple two-zone model for the WD obviously needs improvement, and we have neglected the effects of mixing induced by the breaking gravity waves and viscous angular momentum transport. If the mixing is strong enough to smooth out the WD composition gradients, the dynamics of gravity wave excitation and tidal heat deposition may be altered. Furthermore, if the surface hydrogen mixes into the WD interior where it burns, the surface hydrogen layer will be gradually depleted and a TN will not occur. Observations of the ejecta of classical novae indicate substantial enrichment with core elements, although the mixing mechanism is not well understood (Truran 2002). These and other aspects of TNe in compact WD binaries warrant further study.

Future observations may be able to test whether TNe occur and in turn provide information about the tidal processes at work in WD binaries. The observation of a nova-like event in a system with no evidence for mass transfer would be strong evidence for the existence of TNe and for the tidal heating mechanism studied in this paper. Measurements of hydrogen surface abundances in compact WD systems could also constrain our theory. The observation of a WD with a thick hydrogen envelope in a very tight ( $P \lesssim 5$ minutes) detached binary would indicate that TNe do not usually occur. If WDs in tight binaries are observed to have little to no hydrogen on their surface, this may indicate that TNe have stripped the surface hydrogen, or that the hydrogen has been destroyed due to efficient mixing processes. Observations of compact binary WDs detected in future surveys may provide opportunities to test these theories.

We thank Bill Paxton, Lars Bildsten, and Eliot Quataert for useful discussions. J.F. acknowledges the hospitality (during fall 2011) of the Kavli Institute for Theoretical Physics at UCSB (funded by the NSF through Grant 11-Astro11F-0016) where part of the work was carried out. This work has been supported in part by NSF grant AST-1008245, NASA grants NNX12AF85G and NNX10AP19G.

\section{REFERENCES}

Bloom, J. S., Kasen, D., Shen, K. J., et al. 2012, ApJ, 744, L17

Brown, R. B., Kilic, M., Hermes, J. J., et al. 2011, ApJ, 737, L17

Dan, M., Rosswog, S., Guillochon, J., \& Ramirez-Ruiz, E. 2012, MNRAS, 422, 2417

Di Stefano, R. 2010, ApJ, 719, 474

Fuller, J., \& Lai, D. 2011, MNRAS, 412, 1331

Fuller, J., \& Lai, D. 2012, MNRAS, 421, 426

Gilfanov, M., \& Bogdan, Á. 2010, Nature, 463, 924

Goldreich, P., \& Nicholson, P. 1989, ApJ, 342, 1079

Iben, I., \& Tutukov, A. 1984, ApJS, 54, 335

Iben, I., Tutukov, A., \& Fedorova, A. 1998, ApJ, 503, 344

Li, W., Bloom, J. S., Podsiadlowski, P., et al. 2011, Nature, 480, 348

Loren-Aguilar, P., Isern, J., \& Garcia-Berro, E. 2009, A\&A, 500, 1193

Maoz, D., Sharon, K., \& Gal-Yam, A. 2010, ApJ, 722, 1879

Mochkovitch, R., \& Livio, M. 1989, A\&A, 209, 111

Paxton, B., Bildsten, L., Dotter, A., et al. 2011, ApJS, 192, 3

Piro, T. 2011, ApJ, 740, 53

Raskin, C., Scannapieco, E., Fryer, C., Rockefeller, G., \& Timmes, F. 2012, ApJ, 746, 62

Schaefer, B., \& Pagnotta, A. 2012, Nature, 481, 164

Segretain, L., Chabrier, G., \& Mochkovitch, R. 1997, ApJ, 481, 355

Spruit, H. C. 2002, A\&A, 381, 923

Truran, J. W. 2002, in ASP Conf. Proc. 261, The Physics of Cataclysmic

Variables and Related Objects, ed. B. T. Gänsicke, K. Beuermann, \& K

Reinsch (San Francisco, CA: ASP), 576

van Kerkwijk, M., Chang, P., \& Justham, S. 2010, ApJ, 722, 157

Webbink, R. F. 1984, ApJ, 277, 355

Willems, B., Deloye, C. J., \& Kalogera, V. 2010, ApJ, 713, 239

Yoon, S.-C., Podsiadlowski, Ph., \& Rosswog, S. 2007, MNRAS, 380, 933 